\title{
Design and research of fishing boat accident case library base on spatial database
}

\author{
Yuling Xia ${ }^{1,2}$, Yonghua Sun ${ }^{1,2}$, Wenbin $\mathrm{Li}^{1,2}$, Libin $\mathrm{Qi}^{1,2}$ Cankun Yang ${ }^{1,2}$ \\ ${ }^{1}$ State Key Laboratory Incubation Base of Urban Environmental Processes and Digital Simulation, \\ Capital Normal University, Beijing, 100048, China \\ ${ }^{2}$ College of Resources Environment \& Tourism, Capital Normal University, Beijing, 100048, China \\ email: 2140902038@cnu.edu.cn
}

Keywords: Fishing boat early warning; Artificial immune system; Artificial neural network

\begin{abstract}
The paper builds a model of marine fishing vessel early-warning based on principle of Negative selection algorithm of artificial immune system and Fishing Boat Accident Case Library. Firstly, the index weight of model was calculated by using BP neural network algorithm. Then, the model of fishing vessel early-warning based on artificial immune system was built and tested, through steps of data encoding, affinity calculation, threshold determination, and creating warning detector. $80 \%$ warning success rate proved this model has good warning effect.
\end{abstract}

\section{Introduction}

Marine fishery is the most important industry of the three major marine industries in China which plays an important role in the marine economy. The production safety of the marine fishery is directly related to the interests of the fishermen, which has been the focus of the government departments [1]. In recent years, the coastal provinces have invested a lot of money to build fishing vessels safety monitoring and management system. As the lack of information about the marine environmental factors, which is closely related to the safety of fishery production, it could not make a decision to hedge or rescue, and meet the needs of the growing fishery economy. Therefore the establishment of environmental warning model of marine fishing vessel to prevent marine disasters and reduce economic losses is imperative.

At home and abroad the number of research about the early warning model of marine vessels is small:

T.Ruxtont and J.wang etc. had done a lot of research work on the FSA method [2][3]. Dagang Liu used a comprehensive safety assessment method based on risk analysis technology to analyze the risk system of the navigation in severe weather, and determine the safety of the vessels [4]. Fucai Luo did some research into the risks of fishing vessels, which against sea wind disaster based on a comprehensive safety assessment method [5].

Kunpeng Yang built a safety comprehensive evaluation model of the ship power installation by using artificial neural network algorithm [6]. Zhongyi Zheng used BP artificial neural network to calculate the risk of ship collision [7]. Guofang Chen took the human factors as entry point to establish the risk index system and propose human risk assessment method based on artificial neural network theory [8].

Zhongzhou Fan [9], Xin Li [10], Jie Yao [11] etc. conducted a study to assess the safety of navigation using fuzzy comprehensive evaluation method. Yuqing Ren etc. used set pair analysis method to build vessels' safety comprehensive assessment model [12].

The paper choose the sea wind, waves as environmental early warning indicators, using sea wind, sea waves, fishing positioning data and structural data from the National Marine Environmental Forecasting Center to calculate the weight of the early warning index by optimized BP neural network learning algorithm. And then, a model of marine fishing vessel early-warning is built based on the artificial immune system to provide a theoretical basis for safety management of fishing vessel. 


\section{Artificial immune method}

Inspired by the natural immune system, researchers introduced the idea of immune into artificial intelligence to build artificial immune system model. Artificial immune system has the characteristics of global optimization, tolerance, robustness and adaptability to overcome the shortcoming of other warning model, such as the slow calculation and great subjectivity [13][14][15].

The principle of negative selection algorithm is randomly generate detector, which can detect "Self" be canceled, which can detect "No-self" be retained. The detector is equivalent to antibodies or receptors of the natural immune system in the negative selection algorithm. "Self" refers to the content to be protected, the equivalent of auto-antigen in the natural immune system, such as data, program or normal model. "Non-self" refers to the content which contamination occurred and abnormal change, the equivalent of external antigens in the natural immune system.

In the artificial immune system, Affinity is determined by the degree of matching between "antigen" and "antibody". This paper referencing to existing research results, using a thought of form space, define affinity as the coordinate distance between the antibody and antigen to calculate the affinity. In the form space $S$, a collection of points $x=(x 1, x 2, \ldots, x L)$ may be seen as feature set of antibody - antibodies and antigen - antibody interaction. As shown in Figure 1, form space $\mathrm{S}$ has a region $\mathrm{V}$ ( $\mathrm{V}$ represents the volume of the region). - represents antibodys. $\otimes$ represents antigens. $\varepsilon$ represents threshold, and the antigen can induce antibody immune response within the threshold. Using the decimal coding, the coordinates distance between of antibody and antigen is their affinity as the following formula:

$$
L=(a b-a g)^{2}
$$

Wherein, ab represents the coordinate of warning and monitoring data, and ag represents the coordinate of sample data in Case Library. L represents the distance between antigens and antibodies, and the smaller the value of L, the higher affinity between them.

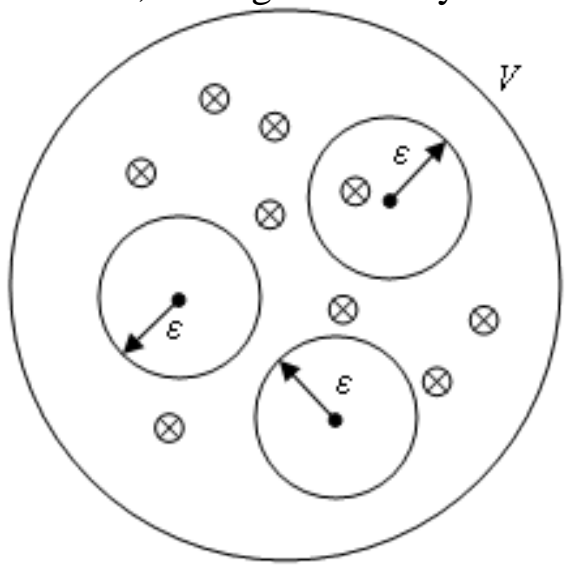

Fig.1. Form space

\section{Artificial neural networks method}

Artificial neural network is the abstract and simulation of human brain. The structure includes input layer, output layer, and the hide layer (as shown in Figure 2). In general, the input layer is mainly used for inputting indicators of sample data; output layer is used for outputting sample calculation results; and the hide layer is used for calculating the index weight. This paper chooses objective weighting method based on $\mathrm{BP}$ artificial neural network to determine the weight of warning index. BP network is a multi-layer feed-forward network trained by error back propagation algorithm, capable of learning and storing a large amount of input-output mode mappings without prior reveal mathematical equations that describe this mapping. Its learning rule is to use the steepest descent method, by reverse spread to constantly adjust the network weights and thresholds, so that square sum of the networks is smallest. 


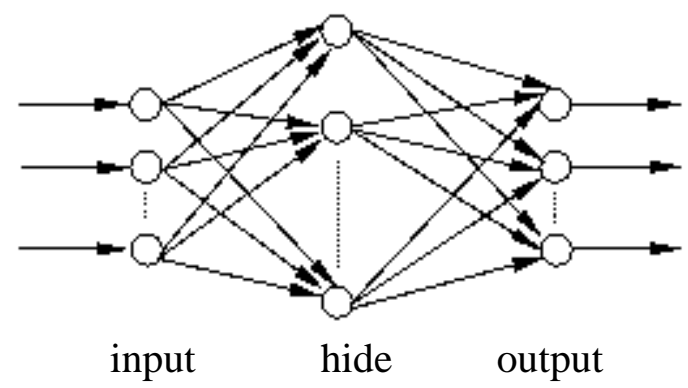

Fig.2. BP neural network structure

\section{Applications}

The data sources are Marine disaster early warning report data and fishing vessel positioning data from National Marine Environmental Forecasting Center. This paper uses the negative selection algorithm to build a warning model of marine fishing vessels (as shown in Figure 3). First, it needs to get the case data of each warning level from Fishing Boat Accident Case Library to build early warning detector, meanwhile obtain real-time positioning data and structure data of fishing vessels to get sea surface waves and sea wind warning data by spatial overlay analysis. Subsequently, prepare for generating early warning detector, it needs to code the sample datum and the case datum. After the completion of data coding, three levels warning detectors (R1, R2, R3) for each warning indicator should be generated using the encoding datum. According to affinity function, the affinity of sample data set and collection of early warning detection is calculated to judge if the affinity is less than a given threshold. If the affinity is less than the given threshold value, it is determined safe state; if the affinity is greater than the threshold, the vessel will receive warning of corresponding level.

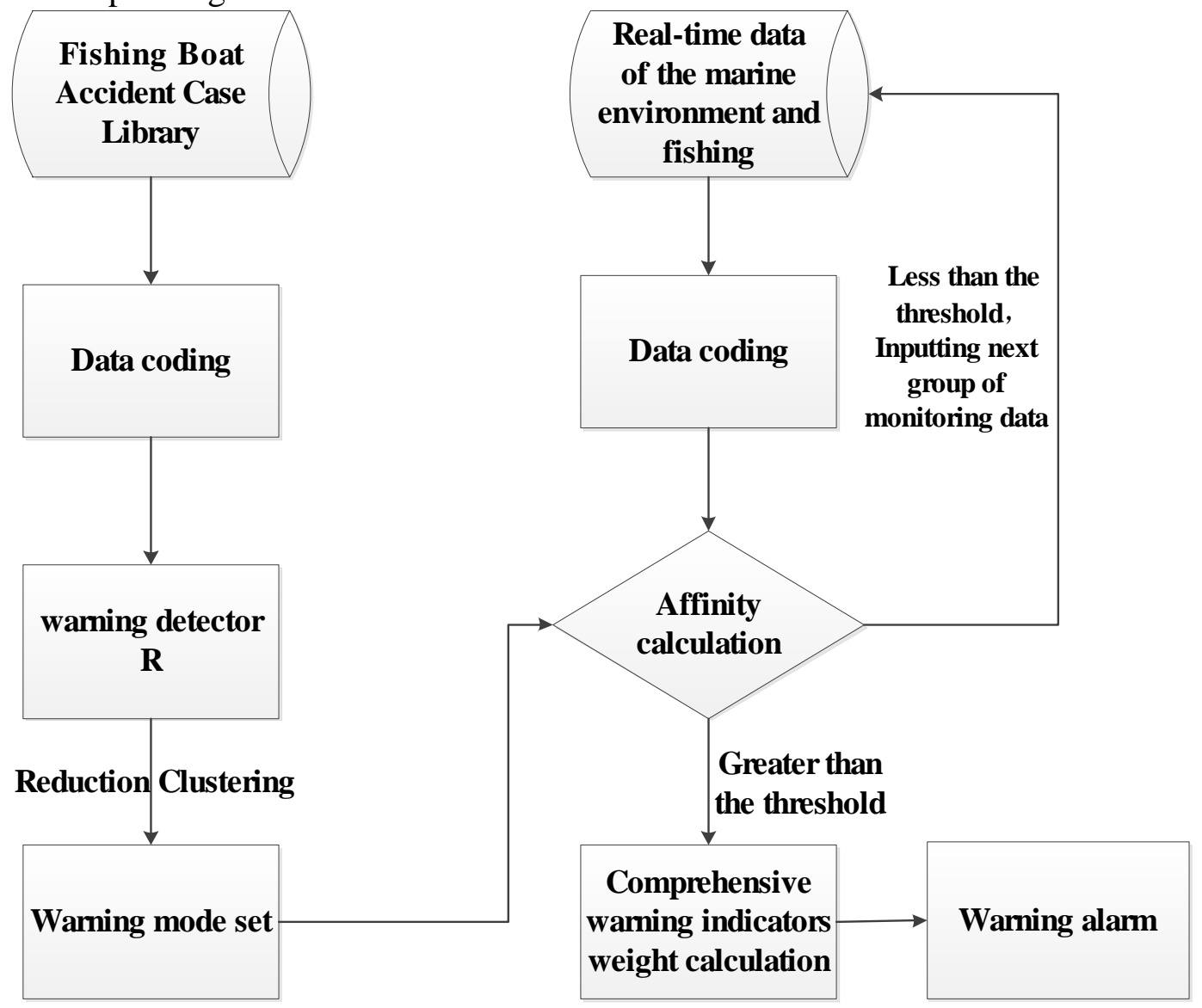

Fig.3. Warning process of Artificial Immune System 


\section{Results}

The paper establish an index system of fishing early warning model including the factors of natural conditions and vessels' structure, based on the Fishing Boat Accident Case Library. The index system is as the input layer neurons; the warning result is as the output layer neurons; and in accordance with relevant weight calculation formula, the corresponding value of the index weight was got in the hidden layer (Results shown in Table 1).

Table.1. Index weight of model

\begin{tabular}{ll}
\hline early warning index & weight \\
\hline fishing vessel tonnage & 0.2052 \\
fishing vessel main motor power & 0.0098 \\
fishing vessel texture & 0.1321 \\
fishing vessel age & 0.1023 \\
sea wind rating & 0.1998 \\
sea wave rating & 0.3508 \\
\hline
\end{tabular}

To prevent interference with warning model calculation, the research selects 10 cases of maritime fishing from Fishing Boat Accident Case Library randomly, and deletes them in the case library. Which in marine accident, the vessels' structure data and sea wave data, as shown in Table 2:

Table.2. data parameter

\begin{tabular}{ccccccc}
\hline $\begin{array}{c}\text { Warning } \\
\text { sample }\end{array}$ & $\begin{array}{c}\text { tonnage } \\
(\mathrm{T})\end{array}$ & $\begin{array}{c}\text { main motor } \\
\text { power }(\mathrm{KW})\end{array}$ & texture & $\begin{array}{c}\text { age } \\
\text { (year })\end{array}$ & $\begin{array}{c}\text { wind } \\
\text { rating }\end{array}$ & $\begin{array}{c}\text { wave height } \\
(\mathrm{M})\end{array}$ \\
\hline sample 1 & 146 & 408 & woodiness & 3 & 5 & 6 \\
sample 2 & 95 & 202 & steel & 19 & 5 & 2 \\
sample 3 & 55 & 132 & steel & 14 & 7 & 4 \\
sample 4 & 35 & 76 & steel & 7 & 6 & 5 \\
sample 5 & 5 & 18 & woodiness & 2 & 9 & 5 \\
sample 6 & 138 & 330 & steel & 11 & 6 & 4 \\
sample 7 & 32 & 79 & steel & 16 & 5 & 4 \\
sample 8 & 21 & 121 & woodiness & 7 & 5 & 4 \\
sample 9 & 4 & 5 & woodiness & 13 & 5 & 3 \\
sample 10 & 1 & 16 & woodiness & 19 & 6 & 4 \\
\hline
\end{tabular}

Data encoded on the sample data, create warning detector $\mathrm{R}$, calculate the affinity between sample data and $\mathrm{R}^{\prime}$ in each police of spatial data, match early warning grade, then according to index weights of ocean fishing warning model, go about a comprehensive early warning calculations. The results are shown in Table 3: 
Table.3. Warning results and the actual results

\begin{tabular}{ccccccccccc}
\hline & sample & sample & sample & sample & sample & sample & sample & sample & sample & sample \\
& 1 & 2 & 3 & 4 & 5 & 6 & 7 & 8 & 9 & 10 \\
\hline $\begin{array}{c}\text { warning } \\
\text { level }\end{array}$ & second & third & third & third & first & third & third & second & third & first \\
$\begin{array}{c}\text { actual } \\
\text { level }\end{array}$ & second & third & third & second & first & first & second & second & third & first
\end{tabular}

As can be seen, only the warning Level of sample 4, sample 6 and sample 7 is different from the actual level in all 10 samples. Warning sample overall success rate reached $70 \%$, it can be seen, the accuracy of ocean fishing warning model is higher.

\section{Conclusion}

Using negative selection algorithm of artificial immune system, the paper builds a new model of marine fishing vessel early-warning based on Fishing Boat Accident Case Library. Finally, the warning results are compared with the actual results. The $80 \%$ success rate proves this model has good warning effects.

\section{Acknowledgement}

In this paper, the research was sponsored by the Special Fund for Ocean Research in the Public Interest (Project No. 201205006-1) and (Project No. 201205017-6).

\section{References}

[1] Faling Lin. Dissicussion of effectively sheltered area for fishery port-An example of Xiaguan fishery port [J]. Marine Forecasts, 2013, 29(6): 92-97.

[2] Wang J, Kieran O. Offshore Safety Assessment and Safety-Based Decision-Making-The Current Status and Future Aspects [J]. Journal of Offshore Mechanics and Arctic Engineering, 2000, 122(2): 93-99.

[3] Wang J. A subjective modelling tool applied to formal ship safety assessment [J]. Ocean Engineering, 2000, 27(10): 1019-1035.

[4] Dagang LIU, Zhongyi ZHENG, Zhaolin WU. Risk analysis system of underway ships in heavy sea [J]. Journal of Traffic and Transportation Engineering, 2004, 4(2):100-102

[5] Fucai LUO, Yan LIN, Yunlong WANG. Fishing vessels safety risk analysis based on formal safety assessment [J], Journal of Dalian Maritime University, 2011,37(2):51-53， 57

[6] Kunpeng YANG, Peng WANG, Xinxiang PAN. Formal safety assessment model of marine power plant based on artificial neurual network [J]. Journal of Safety Science and Technology, 2006, 2(6s): 90-93

[7]Zhongyi Zheng, zhaolin Wu. With BP neural network calculation of ship collision risk [J]. World Shipping, 2000, 23(2): 4-6.

[8] Guofang Chen,Yan Liu,Baozhi Chen. Human risk assessment based on neural network BP algorithm [A]. Chinese Science and Technology Institute of Labor Protection,2003,4.

[9] Zhongzhou Fan. Assessment on the mathematics model for the safety of bulk carrier [D]. Dalian Maritime University, 2002.

[10] Xin Li. The research and evalution on navigation safety of Ro-Pax vessel in the water area of Bohai bay [D]. Dalian Maritime University, 2003. 
[11] Jie YAO, Yuqing REN. Study on the evaluation method of fishing vessel's sailing safety [J]. Journal of Dalian Fisheries University, 2005, 20(3): 244-248.

[12] Yuqing Ren, Jihui Zheng, Hui Dong. A comprehensive evaluation model of fishing vessel safety based on set pair analysis [J]. Navigation of China, 2012, 35(2): 60-63.

[13] Mingfeng Wu. Research on the Model of Urban Ecosystem Early-Warning Based on Artificial Immune [D]. NanJing: HoHai University, 2006.

[14] Junfei Chen. Research on Urban ecosystem Monitor and Early-Warning [D]. HoHai University, 2005.

[15] Minhui Wang, Lei Qiu. Diagnosis and Warning of REE Complex System [M]. BeiJing:Science Press,2007 